\title{
Identification of Instability Modes of Transition in Underexpanded Jets
}

\author{
Jennifer A. (Wilkes) Inman*, Paul M. Danehy ${ }^{\dagger}$, Robert J. Nowak ${ }^{\ddagger}$, and David W. Alderfer ${ }^{\S}$ \\ NASA Langley Research Center, Hampton VA, 23681-2199
}

\begin{abstract}
A series of experiments into the behavior of underexpanded jet flows has been conducted at NASA Langley Research Center. Two nozzles supplied with high-pressure gas were used to generate axisymmetric underexpanded jets exhausting into a low-pressure chamber. These nozzles had exit Mach numbers of 1 and 2.6, though this paper will present cases involving only the supersonic nozzle. Reynolds numbers based on nozzle exit conditions ranged from about 300 to 22,000 , and nozzle exit-to-ambient jet pressure ratios ranged from about 1 to 25 . For the majority of cases, the jet fluid was a mixture of $99.5 \%$ nitrogen seeded with $0.5 \%$ nitric oxide (NO). Planar laser-induced fluorescence (PLIF) of NO is used to visualize the flow, visualizing planar slices of the flow rather than path integrated measurements. In addition to revealing the size and location of flow structures, PLIF images were also used to identify unsteady jet behavior in order to quantify the conditions governing the transition to turbulent flow. Flow structures that contribute to the growth of flow instabilities have been identified, and relationships between Reynolds number and transition location are presented. By highlighting deviations from mean flow properties, PLIF images are shown to aide in the identification and characterization of flow instabilities and the resulting process of transition to turbulence.
\end{abstract}

\section{Nomenclature}

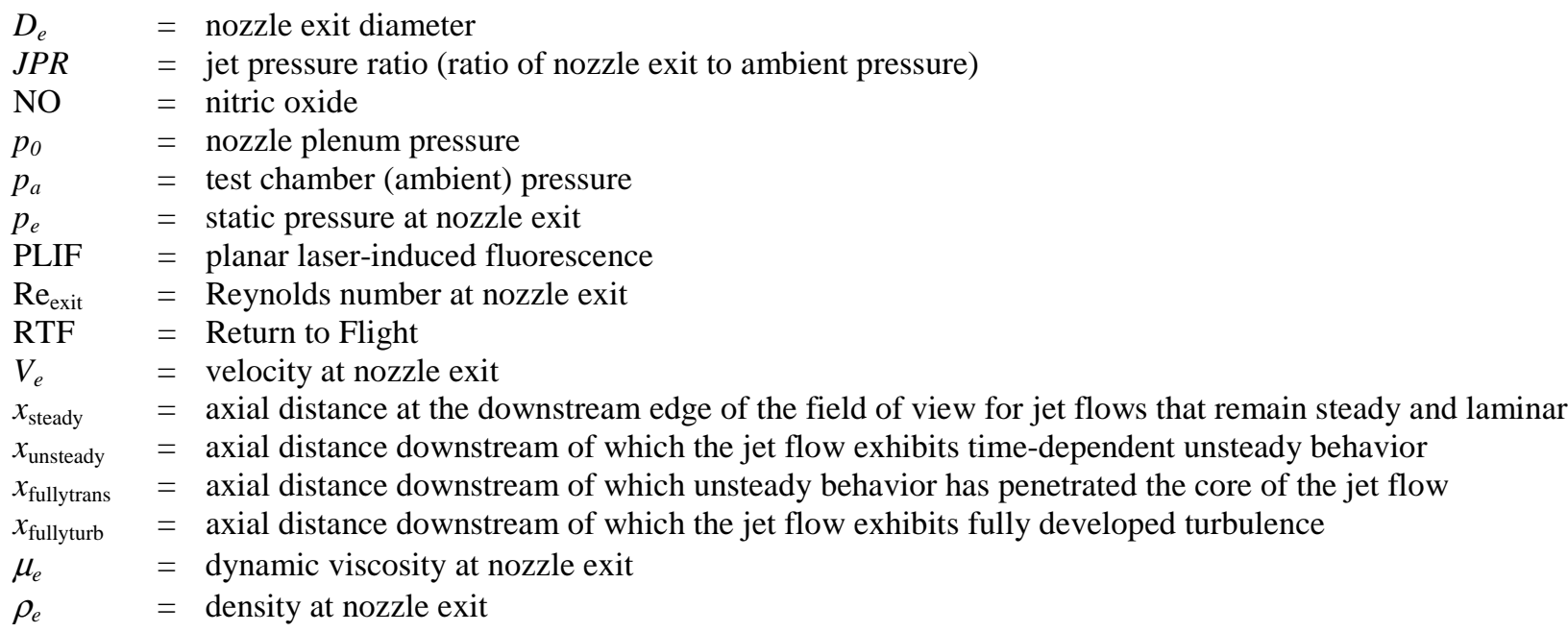

\section{Introduction}

T $N$ the wake of the loss of the Columbia orbiter due to a breach in the leading edge of its left wing, a series of tests 1 were conducted in an effort to better understand the flowfields resulting from breaches in the outer structure of reentry vehicles. Penetration of hot gas through breaches could impact internal structures, causing failure of the

\footnotetext{
${ }^{*}$ Research Scientist, Advanced Sensing and Optical Measurement Branch, MS 493, AIAA Member.

${ }^{\dagger}$ Research Scientist, Advanced Sensing and Optical Measurement Branch, MS 493, AIAA Associate Fellow.

¥ Research Scientist, Aerothermodynamics Branch, MS 408A, AIAA Member.

${ }^{\S}$ Research Scientist, Advanced Sensing and Optical Measurement Branch, MS 493.
} 
vehicle. These tests were conducted in support of the Orbiter Aerothermodynamics Working Group as part of NASA's Shuttle Return to Flight (RTF) effort. The tests involved simulating flow through a hypothetical breach in the leading edge of the Space Shuttle Orbiter along its reentry trajectory, with the goal of studying the transition to turbulence in such flows, as well as generating a data set with which other researchers can test and validate computational modeling tools. A subset of these tests used planar laser-induced fluorescence (PLIF) of nitric oxide (NO) to visualize the flow issuing from a nozzle into a low-pressure chamber. We have previously reported the use of PLIF to investigate both steady and unsteady underexpanded free (i.e. non-impinging) jets issuing from a sonic nozzle. ${ }^{1}$ Three steady, laminar cases were compared with computational fluid dynamics (CFD) in another paper. ${ }^{2}$ A third paper focused on steady, laminar, impinging jet flows. ${ }^{3}$ A future paper will present results for unsteady jets impinging on a flat plate, in regards to the effect of jet impingement upon the process of transition to turbulence. This paper focuses upon the transition to turbulence in free jet flows issuing from a supersonic nozzle with a nominal exit Mach number of 2.6.

Unsteady-laminar, transitional, and turbulent free jet flows will be covered in this paper. It will introduce the concept of unsteadiness, including the underlying flow properties of viscosity and vorticity. It will explain how viscosity plays a dual role, both as a stabilizing and destabilizing force. It will give an overview of the sources/origins of vorticity in a flow, namely, non-uniform flow velocity. Several primary sources of free jet instabilities will be identified. These include the nozzle lip, shock intersections, shear layers, and flow maxima (defined as flow locations where the jet diameter is locally a maximum-and pressure is locally a minimumcompared to the diameter of the jet immediately upstream and downstream of that location. Also referred to as antinodes in contrast with their high-pressure counterparts, called nodes). It will be shown that flows with a distinct high-velocity jet boundary have different modes to transition than do those with an oscillating flow structure. The concept of longitudinal (also called axial or streamwise) and azimuthal normal modes will be introduced as well, though in a limited capacity (here, normal refers to the fact that these modes are orthogonal to one another). Two primary longitudinal modes will be considered - the sinuous (antisymmetric) and varicose (symmetric) modes. A brief introduction to azimuthal (cosine) modes will be given, along with some reconstructed flow cross-sections showing flows with active azimuthal modes.

The difficult task of accurately predicting the transition from laminar to turbulent flow remains an unsolved problem for many ranges of conditions. Furthermore, CFD (computational fluid dynamics) turbulence models are generally based upon boundary layer flow along surfaces rather than free shear flow, and are often based on data taken in the incompressible (with a Mach number less than about 0.3 ) regime ${ }^{4}$, whereas the present experiments are in the compressible flow regime. The measurements of the unsteady, transitional, and/or turbulent locations resulting from these tests can therefore potentially be used to determine where turbulence models should be implemented in CFD simulations as well as providing data for comparison with CFD results.

We have developed an image processing technique, involving calculation of the standard deviation of the intensity in PLIF images in order to aid in the identification of turbulence, with the aim of empirically determining criteria governing the onset of turbulence. Jet scaling parameters were used to define a rescaled Reynolds number that incorporates the influence of a varying pressure ratio. The image processing procedure that we have employed will be described, as well as an explanation of the standard deviation images and how they can aid in the identification of flow unsteadiness, transition, and turbulence. Finally, measurements of the axial location associated with various stages in the transitional process will be presented for all supersonic free jet cases.

\section{Experimental Description}

\section{A. Facility and Hardware}

Tests were conducted at NASA Langley Research Center using the test section of the 15-Inch Mach 6 Wind Tunnel as a vacuum chamber. Nitrogen or helium seeded with $0.5 \%$ nitric oxide was plumbed into a heated stainless steel plenum, through a nozzle, and into the vacuum chamber. Two different nozzles were used: the first-a converging nozzle with the exit at the smallest diameter, or throat, and hereafter referred to as the sonic nozzle—had a nominal exit Mach number of 1; the second-a converging/diverging nozzle, hereafter called the supersonic nozzle-had a nominal exit Mach number of 2.6. Mass flow controllers controlled the flow rates, which also indirectly controlled the plenum pressure upstream of the nozzle. Further details can be found in Refs.1-5 


\section{B. Test Parameters}

For each hardware configuration, two flow parameters were varied: the exit Reynolds number $\left(\operatorname{Re}_{e x i t}\right)$ and the jet pressure ratio $(J P R)$. $\operatorname{Re}_{e x i t}$ was defined in terms of the nozzle exit diameter, $D_{e}$, and the density $\rho_{e}$, velocity $V_{e}$, and dynamic viscosity $\mu_{e}$ at the nozzle exit, as given by (1).

$$
\operatorname{Re}_{e x i t}=\frac{\rho_{e} V_{e} D_{e}}{\mu_{e}}
$$

$\mathrm{Re}_{e x i t}$ was affected by nozzle plenum temperature and was varied by changing the mass flow rates. JPR was defined as the ratio of the calculated static pressure at the nozzle exit, $p_{e}$, to the ambient pressure in the test section, $p_{a}$, according to (2), and was varied by changing the test section pressure for a given Reynolds number (and therefore, a fixed $p_{e}$ ).

$$
J P R=\frac{p_{e}}{p_{a}}
$$

\section{PLIF Flow Visualization Technique}

The PLIF laser system includes a tunable Nd:YAG-pumped dye laser followed by doubling and mixing crystals. The resulting output, at $226.256 \mathrm{~nm}$, was tuned to excite the strongly fluorescing spectral lines of NO near the $\mathrm{Q}_{1}$ branch head ( $\mathrm{Q}$ denotes a change in rotational quantum number equal to zero). Optics formed the beam into a laser sheet that was $100 \mathrm{~mm}$ wide $\mathrm{x} \sim 0.2 \mathrm{~mm}$ thick (FWHM) in the measurement region. Fluorescence was imaged onto a gated, intensified CCD at a viewing angle normal to the laser sheet. Images were acquired at $10 \mathrm{~Hz}$ with a $1 \mu \mathrm{s}$ camera gate and a spatial resolution of between 3 and 7 pixels $/ \mathrm{mm}$, depending on the desired field of view for a given test condition. The laser beam could be scanned spatially through the flow using optics attached to a translation stage to visualize different cross-sections of the flow.

\section{Terminology: Unsteady, Transitional and Turbulent}

Here, the term laminar is used to indicate flows that are smooth, that have structures that are not time-varying, that have no vortices, and that are not transitional or turbulent. Unsteady laminar flows still lack turbulent structures, but do exhibit time-dependent fluctuations. Such fluctuations may be periodic or chaotic, but the structures associated with these fluctuations are inherently dependent upon the geometry of the flow, and are generally of a spatial scale comparable to the scale of the flow structures of the underlying flow. Transition, by contrast, is characterized by flow structures that progressively break down from larger scales to smaller scales, with energy being redistributed to increasingly smaller spatial scales as the flow becomes highly unsteady. Fully developed turbulence is the limiting case of this process.

In a fully developed turbulent flow, the local flow structures bear little memory of the larger flow from which they originated, are theoretically homogeneous and isotropic (that is, are statistically invariant under translation and rotation, respectively - a condition that never perfectly exists in physical flows), and are generally of a much smaller scale, down to the molecular dissipation scale (also called the Kolmogorov scale). For an in-depth discussion of the inherent ambiguities and difficulties involved in developing a precise definition for each of these categories, see the comprehensive texts by Pope, ${ }^{6}$ Drazin and Reid, ${ }^{7}$ and Lesieur. ${ }^{8}$

Even though many previous studies have made some measurement of turbulence-using, for instance, hotwire anemometry ${ }^{9,10}$ - very few studies of supersonic free jets discuss turbulent flow structures. Notable exceptions include the free-jet studies of streamwise vortices in the outer shear layer of underexpanded jets by Krothapalli et al. ${ }^{11}$ and Zapryagaev et al. ${ }^{12}$ Krothapalli et al. successfully visualized cross-sectional slices through the flow using Mie scattering off of moisture in the ambient air that condensed when mixed with the cold fluid of the jet. In the study by Zapryagaev et al., the presence of these vortical flow structures was inferred from Fourier spectral analysis of pitot pressure surveys, being the most likely explanation for the measured azimuthal pressure variations. A major contribution of the present work is that the flow structures associated with the onset of unsteadiness and through the transitional, developing regimes have been visualized directly.

\section{E. Identification of Unsteady Flow Behavior Using PLIF Images}

\section{Image Processing}

The image processing procedures that have been used for the PLIF images presented herein are described more fully in Refs. 1, 3, and 5. An overview is given in the following sections. After a background image is subtracted from each smoothed PLIF image, significant left-to-right variations in intensity remain. These variations are due to the left-to-right variations in both the laser sheet intensity and laser wavelength. To correct for these effects, the 
images are divided by a laser sheet profile. As final steps in the image processing procedure, images were sometimes cropped, a mask was applied to cover the nozzle hardware in order to eliminate any persistent glints or scattered light, and a false-color mapping was applied. The spatial resolution was determined by imaging either a ruler (in earlier tests) or a dotcard (in later tests) positioned in the same plane as the laser sheet. The optical access in these experiments permitted perpendicular viewing of the measurement plane and no significant perspective or lens distortion was found in the images. For experimental configurations where optical access is more limited and perpendicular viewing is not possible, dotcard imaging makes it possible to correct for perspective and lens distortion. ${ }^{13}$

\section{Standard deviation images}

After background subtraction and laser sheet correction, an average image was created from all the images from a given run (each run consisting of either 100 or 200 single-shot images). A standard deviation image was then created in the following manner. Each single shot image was subtracted from the averaged image. The images contained intensities spanning a large dynamic range, and so the difference in intensity was divided by the average intensity, resulting in a percentage difference, rather than an absolute difference. This percentage difference in intensity at each pixel was squared, and the squares were summed over all images in the run. Finally, the sum of the squares was divided by the number of images and the square root was taken. The resulting image provides a map of the flow, highlighting regions of large percentage variations in intensity. In general, steady laminar flows will have relatively consistent shot-to-shot intensity at a given location in the flow, resulting in percentage standard deviations of less than about $30 \%$. Unsteady flows, by contrast, will have regions where the intensity varies in each image, resulting in typical percentage standard deviations of between about $35 \%$ and $100 \%$. A drawback of this technique is that it tends to more prominently highlight variations in regions where there are steep gradients in image intensity in the average image (e.g. along jet boundaries).

\section{Identification of changes in flow state}

Conceptually, a given flow, or parts of a flowfield, can be divided into four types of flow-steady laminar, unsteady laminar, transitional, and turbulent, not all of which will necessarily be present under a given set of conditions. The standard deviation images described above have been used to distinguish between each of these flow types. For simplicity, we have defined four regions (based on these four flow types) as a function solely of the axial coordinate, $x$. This means, for example, that in the region we have called the unsteady laminar region, the core of the jet may still be unsteady laminar, while the edges of the jet are exhibiting transitional behavior. The criteria that have been used to define the boundaries between these regions are defined as follows.

$x_{\text {steady }}$ - If the flow is entirely steady laminar within the field of view, the furthest downstream location is marked as $x_{\text {steady. }}$ In standard deviation images, this means that the intensity remains low and relatively constant across the image. An example of a steady laminar flow is given in Fig. 1.

$x_{\text {unsteady }}-$ This is defined to be the downstream location at which the flow first exhibits unsteady behavior. All locations upstream of this point are thought to be steady laminar flows; all locations downstream have some level of unsteadiness to them. In standard deviation images, this location is characterized by an increase in intensity above the background (noise) level. Figure 2 shows a flow exhibiting unsteady laminar behavior. In Ref. 1, this distance was labeled $x_{\text {trans. }}$. Although some transitional behavior may be observed in the region we are calling laminar unsteady, especially along the boundaries of the jet, in the present work we have elected to wait to label a flow as transitional until the entire flow is transitional.

$x_{\text {fullytrans }}-$ This is defined to be the location at which all of the jet, including the jet core, is exhibiting transitional behavior. In standard deviation images, it is the location where the intensity in the core of the flow has risen above the background level. Figure 3 shows such a case. This distance is similar to what was labeled the turbulent distance in Ref. 1.

$x_{\text {fullyturb }}-$ This is defined to be the location beyond which the flow can be said to be fully developed. In other words, all spatial modes are active, down to the smallest scales. This location is characterized by a decrease in the intensity in the standard deviation images relative to the peak transitional intensity. Figure 4 illustrates a run exhibiting fully developed turbulence. Prior to this point, "turbulent" structures may be seen, but the smaller scales may be missing. Once the flow is fully developed, however, it exhibits increased homogeneity (and thus, the lower standard deviation in intensity), with widespread small-scale structures.

\section{Volume Imaging}

In addition to data taken along the flow centerline, volumetric imaging was performed for many of the test cases. To obtain these data, a series of 200 single-shot images was acquired as the laser sheet was swept spanwise through the flow, providing slices of the flow field. These slices allow the reconstruction of cross-sections of the flow in planes perpendicular to the jet axis, a technique which will be referred to as volume imaging. The single-shot 
images acquired on the flow centerline during the spatial scan of the laser sheet provide a reference for the relative locations of these slices.

Slices reconstructed from scans through unsteady flows could potentially be used to give an indication of the level of unsteadiness at a given axial location. For the relatively steady upstream slice location, the flow appears to be a smooth circle. As slices are reconstructed further downstream, the circular outline of the flow becomes jagged, due to shot-to-shot variations in the position of the jet. Thus, a single slice image can provide a visual representation of the degree of flow unsteadiness. Volume imaging also represents a promising means of making PLIF measurements in flows where optical access constraints do not permit imaging in the most desirable measurement plane.

\section{F. The Dual Nature Viscosity}

Vorticity is a measure of the amount of rotation in a flow. Flows lacking vorticity are said to be irrotational. Vorticity plays a crucial role in the development of turbulence. Mathematically, vorticity is defined to be the curl of

the velocity: $\vec{\Omega}=\nabla \times \vec{v}$. The viscosity of the flow sets up a velocity gradient across the boundary between a jet and the ambient fluid, resulting in a region called a shear layer. In highly-underexpanded jet flows, there are two such shear layers. Figure 5 is a PLIF image of an underexpanded jet issuing from a sonic nozzle with major flow structures identified, including these two shear layers. The outer shear layer lies between the jet boundary and the ambient gas. The inner shear layer lies between the high-velocity gas near the jet boundary and the slip line emanating from the triple point and separating the high velocity gas from the jet core. Interestingly, then, it is actually the lack of viscosity—or rather, the lack of sufficient viscosity—that results in the flow developing vorticity. That is, if the flow was fully viscous, momentum would be transferred to the surrounding fluid and velocity field would rapidly become uniform, with little or no vorticity, until the flow encountered a wall or other solid boundary.

Viscosity can be described as measure of the resistance of a fluid to deform under shear stress, a measure of the internal resistance of a fluid to flow, and a measure of the internal friction of the fluid. ${ }^{14}$ In a fluid with low viscosity, the fluid is easily deformed and readily flows in different directions in response to applied or internal forces. Flows with high viscosity, by contrast, will resist applied forces to some extent. In this manner, viscosity acts as a type of damping force, and is thus a stabilizing force that counteracts small perturbations of a flow. It can decrease the internal vorticity of the flow by reducing velocity gradients, and thus can dampen out turbulent flow structures.

In addition to acting as a stabilizing force, viscosity can also act as a destabilizing force. Viscosity can introduce velocity gradients - and therefore, vorticity —into regions of a flow that would otherwise be irrotational. If velocity gradients are present in a flow (the slip line between the ambient gas and the high-velocity jet boundary is a perfect example in the jet flows under investigation), viscosity acts to increase the velocity of the slower fluid and decrease the velocity of the faster fluid. Thus, the velocity gradient that would have been a spatial step function in an inviscid fluid acquires a finite spatial extent in a viscous fluid. That is, viscosity causes the velocity gradient that existed at the jet boundary to establish the outer shear layer, expanding the extent of the region containing velocity gradients, potentially all the way to the core of the jet. Given the existence of these competing processes, whether or not a given flow actually goes turbulent depends on which of the possible mechanisms ultimately dominates.

\section{Experimental results}

The jet flows issuing from the nozzles used in these tests enter the test section as laminar jets. Perturbations to these flows are introduced by random fluctuations in the flow or by viscous effects, as described below. Such perturbations may be damped out or may be amplified. The following sections describe the insight into this process that was gained by visualizing jet flow using PLIF and discuss the likely origins of these perturbations.

\section{A. Characteristic Normal Modes of Instability}

Instabilities can be broadly categorized in terms of the normal (i.e. orthogonal) modes of a given jet flow.

One way to model turbulent jet flow is to first model the underlying stable flow, and then introduce perturbations to this base flow. For jets issuing from an axisymmetric nozzle, the perturbations can be either axial (longitudinal) or azimuthal. All possible perturbations to a flow can theoretically be modeled as linear combinations of a complete set of normal modes. ${ }^{15}$

The axial (or longitudinal) normal modes are of two types-symmetric and antisymmetric. ${ }^{16}$ Symmetric modes consist of axially symmetric expansions and contractions of the jet around its axis. These modes are known as varicose modes (also called the sausage instability ${ }^{7}$ ). Figure 6 illustrates a flow in which a varicose mode has 
become active. Identification of unsteady regions of the flow has been enhanced by using the image processing technique described in section II.E. Large standard deviations are indicative of a high degree of unsteadiness in the flow.

Antisymmetric modes consist of sinusoidal oscillations or flapping around the jet axis. These modes are known as sinuous modes, ${ }^{7}$ and they break the axisymmetry of the flow. Figure 7 shows an example of a jet exhibiting the sinuous mode.

The other types of normal modes are azimuthal modes. They have some symmetry around the jet axis, though they are not purely axisymmetric. Mathematically, this symmetry is of the form $\cos (m \theta)$, where $m$ is an integer and $\theta$ is the angle in cylindrical coordinates. The only axially symmetric azimuthal mode is the trivial, non-perturbative $m=0$ mode. Figure 8 shows the geometry of azimuthal modes for several values of $m$. Figures 9 and 10display cross-sectional slices at six axial locations. In both of these figures, the axial symmetry of the jet is seen to be breaking down as azimuthal cosine modes become manifest. While the centerline image in Fig. 10 shows some evidence of this breakdown, no such evidence can be seen on the centerline in Fig. 9. This demonstrates one of the advantages of this technique, since it can reveal non-axisymmetric flow structures that may not be evident in centerline imaging.

When modeling these flows, the jet can be considered as being perturbed by all of these instability modes. As the modes compete with each other, the most dominant mode eventually takes over. A given jet flow will either amplify or dampen the growth of each normal mode of instability. Thus, a particular jet flow may be stable against one type of disturbance, but unstable against others. Additionally, some flows may be positively stable against small perturbations but unstable if the perturbations exceed some critical amplitude. The basic flow may be positively stable, neutrally stable, or negatively stable (unstable) against these perturbations. That is, these disturbances may be damped out, may persist, or may be amplified, respectively. Because most experimental investigations of turbulence have used hotwire anemometry measurements, most of the mathematics that has been developed actually deals with temporal evolution of these disturbances. The present investigation using PLIF is more concerned with the spatial distribution and propagation of these disturbances. There also are the peculiar restrictions on propagation of instabilities (or of any change, for that matter) in the supersonic regions of the flow, since "information" (anything that travels by pressure wave) can only propagate in the downstream direction.

In Hydrodynamic Stability, ${ }^{7}$ Drazin and Reid indicate that at first, one instability mode is active, and then other modes become active in turn. This happens at an ever-increasing rate (spatially, this would meant that the distance from the onset of the first mode to the onset of the second mode would be longer than, say, the distance between the onset of the second mode and the onset of the third mode). Finally, at the onset of fully-developed turbulence, all modes are active. This results in flow structures of all scales from the scale of the flow down to molecular scales.

\section{B. Sources of Vorticity and Growth of Instabilities}

In agreement with our prior work on sonic jets, ${ }^{1}$ the present investigation found that the process of transition from laminar to turbulent flow was a function not only of jet Reynolds number, but also of jet pressure ratio. This pressure ratio dependence was found to occur largely due to the influence on jet geometry and the changes in flow structure associated with changes in jet pressure ratio. Several types of flow structures were identified as contributors to the growth of jet instabilities, which led to transition onset in some cases. Examples of each type of flow structure, including flow near the nozzle exit, shock intersections, shear layers, and locations (antinodes) where the diameter of the jet reaches a local maximum, are shown below.

1. Effect of Reynolds number

The Reynolds number is often used as a gauge of the susceptibility of fluid flow to become turbulent. At low Reynolds numbers, viscous forces dominate and the flow is expected to remain laminar. At high Reynolds numbers, momentum forces are greater and flows tend to become turbulent. As expected, the flows in the present investigation showed a strong dependence of transition location on Reynolds number. Figure 11 shows an example of several cases with the same JPR for a range of Reynolds numbers.

The figure shows single-shot images from six runs with similar jet pressure ratios but different exit Reynolds numbers. Clearly, the onset of transition to turbulence occurs earlier for higher Reynolds numbers, and not at all for the lowest Reynolds numbers.

2. Effect of the jet pressure ratio

An unexpected finding of this experimental program was that the transition location is somewhat dependent upon the jet pressure ratio and not just the Reynolds number. The reason for this dependency seems to stem from the different flow structures that exist in flows of different JPR. General classes of flows exist for the nozzles and range of pressures that were studied, including diamond shock patterns and barrel shock structures. Transition to turbulence can be understood to result from the growth of instability modes within the flow. ${ }^{7}$ The turbulent mode 
that experiences the greatest amplification will depend strongly upon the underlying flow structure. As the Reynolds number is increased for a steady flow with a spatially periodic structure, the unstable modes of the flow will be different than those of, for example, a large barrel shock with a high-velocity jet boundary that is parallel to the axis of the jet. These modes are discussed in greater detail in the following section.

3. Inner and outer shear layers

If a jet structure has a discernible Mach disk, the intersection of the oblique shock (which emanates from the triple point) with the jet boundary, and the triple point itself both seem to serve as a source of instabilities. Figure 12 shows an example of a run where this was the case. Sometimes, slight instabilities are evident in the jet boundary prior to these shock intersection points, but they seem to grow relatively slowly around the barrel shock. Notice the small increases in intensity around the barrel shock (compared to the background intensity level) in the standard deviation image. Prior to the Mach disk/triple point, the gas that has been processed by the oblique barrel shock is bordered to the outside (along the free jet boundary) by slower (stagnant) ambient fluid. To the inside, it is bordered by the faster moving gas inside the barrel shock. So for the gas surrounding the barrel shock, the flow experiences the stabilizing influence of acceleration from inside, but the destabilizing influence of deceleration from the outside.

Then notice the increase in intensity in the inner and outer shear layers (starting at the triple point and reflected shock/jet boundary intersection, respectively). There are many steep velocity gradients in the triple point region. At the Mach disk, this faster-moving gas inside the barrel shock is abruptly decelerated to subsonic speeds. The gas that flows through the reflected shock emanating from the triple point is now bordered both to the inside and to the outside by slower-moving fluids. As a result, this gas becomes the fastest-moving part of the flow downstream of the triple point. This high velocity jet boundary is bordered to the inside and outside by shear layers. After the triple point, both the inner and outer regions of the flow have a destabilizing effect on the gas processed by the barrel shock, since they both cause the high velocity gas to decelerate. The triple point is the meeting point of four different velocity regions - inside the barrel shock (where the Mach number is greater than one), in the jet boundary upstream of the oblique shock (where the Mach number is also greater than one), in the high-velocity jet boundary downstream of the oblique shock, and in the jet core, after the Mach disk. The intersection of the shock emanating from the triple point with the free jet boundary is, in effect, surrounded by three regions of different velocities-the jet boundary before the oblique shock, the high-velocity jet boundary after the oblique shock, and the free stream/ambient gas. Such large velocity gradients imply regions of significant vorticity, and thus regions where instabilities are introduced and/or amplified.

4. Flow maxima (low-pressure antinodes)

If no Mach disk is present, then instabilities seem to originate in the jet boundary at a flow maximum/antinode, just as the flow stops expanding and begins to converge again. Examples of two such runs are given in Fig. 13. Flow maxima are regions of curvature where fluid has been pulled away from the core flow, versus minima (nodes), which also have large curvature, but where the momentum of the flow has compressed it back toward the core of the flow. The flow accelerates when it is expanding, but decelerates when it is converging. As indicated below, acceleration tends to be a stabilizing mechanism, whereas deceleration tends to be a destabilizing mechanism.

At a flow minimum, the core of the flow begins expanding outwards toward the boundary of the jet where instabilities are likely to exist. This expansion may provide a stabilizing influence on the flow. At a flow maximum (low-pressure antinode), however, unstable fluid elements may simply travel along streamlines that are closer to straight than the high-curvature streamlines of stable fluid elements. At this point, the momentum forces are potentially becoming commensurate with the viscous forces, and beginning to exceed them. In this way, the local Reynolds number may be increasing to above a critical value. (Fluid mechanical literature often talk about the critical Reynolds number, $R e_{\mathrm{c}}$, above which a flow transitions. For example, in fluid flow through a pipe, $R e_{\mathrm{c}}$ is on the order of $2,000 .^{8}$ )

\section{The destabilizing influence of deceleration}

According to Drazin and Reid, ${ }^{7}$ decelerations are more destabilizing than accelerations, in general. Accelerations and the associated pressure gradients tend to stretch the flow, whereas decelerations tend to make it crumple or fold back upon itself. The stretching of acceleration tends to oppose vortex/eddy formation, whereas the crumpling effect of deceleration tends to increase the vorticity of the flow and serves as a source of eddy formation. Most regions of these jets are decelerating. Exceptions include expansion regions, such as the region inside a barrel shock, as well as the jet core downstream of a Mach disk, where the inner (slip line) shear layer accelerates the core flow that had been slowed down by the normal shock wave. In the repeating diamond shock pattern, there are repeated expansions (accelerations) and contractions (decelerations). As shown above, for supersonic jets with an oscillating flow structure, transition often begins on the compression side of such an oscillation, where the diameter of the flow is a locally a maximum.

American Institute of Aeronautics and Astronautics 092407 


\section{Flow structures that introduce vorticity}

Based on the measurements presented below, there appear to be three primary locations or flow structures that serve as sources of instability, or at least serve as sources of instability amplification. The first of these is the lip of the nozzle exit; the second is locations of shock wave intersections or reflections, such as the triple point that exists in flows with a barrel shock structure; the third are flow maxima/antinodes that occur in flows with a spatially oscillating structure. Instabilities were not observed inside the supersonic portion of the barrel shock structure for any flow conditions. At most, the position of the Mach disk itself may fluctuate. Instead, for flow with a Mach disk, turbulent features always appear first in the inner and outer shear layers adjacent to the high-velocity jet boundary.

\section{Measured Transition Lengths}

For each test condition, the standard deviation image and the single-shot images were examined. The flow was then labeled steady laminar, unsteady, fully transitional, or fully turbulent according to the criteria defined in section II.E. Note that these locations were determined by eye, and so involved a measure of subjectivity.

As reported in Ref. 1 for sonic nozzle results, when measured distances to transition were graphed in terms of Reynolds number alone, no obvious correlation was observed. This led to the conclusion that jet behavior was a function not only of Reynolds number, but of jet pressure ratio as well. This same conclusion was extended to supersonic nozzle results.

Scaling laws described by Yüceil and Ötügen ${ }^{17}$ provide a means of redefining the Reynolds number to include the effects of JPR. Conditions at the nozzle exit serve as initial conditions for defining an equivalent jet. They assume that the initial jet is allowed to expand adiabatically until the pressure in the jet reaches the ambient pressure. At this point - which they call location 2-all of the flow conditions are recalculated. The expressions for rescaled density $\rho_{2}$, velocity $V_{2}$, temperature $T_{2}$, and jet diameter $D_{2}$ can be found in Refs. 5 and 17 . These yield an expression for the rescaled Reynolds number in terms of $J P R$ and nozzle exit conditions. This expression is given in Eq. 3:

$$
\begin{gathered}
\operatorname{Re}_{2}=\frac{\rho_{2} V_{2} D_{2}}{\mu_{2}}=\operatorname{Re}_{e x i t} \frac{\rho_{2}}{\rho_{e}} \frac{V_{2}}{V_{e}} \frac{D_{2}}{D_{e}} \frac{\mu_{e}}{\mu_{2}} \\
=\operatorname{Re}_{e x i t} \frac{\left(\gamma M_{e}^{2} J P R+J P R-1\right)^{1 / 2}\left(2^{1 / 2} \gamma^{2} M_{e}^{2} J P R^{2}\right)^{n}}{\left.2 \gamma M_{e}^{2} J P R(\gamma+J P R-1)-(\gamma-1)(J P R-1)^{2}\right]^{n+1 / 2}} .
\end{gathered}
$$

The correlation data for the supersonic nozzle cases show different trends than the sonic nozzle data reported in Refs. 1 and 5. Figure 14 shows a plot of rescaled Reynolds number, $R e_{2}$, versus nondimensionalized downstream distance. For these data, a clear Reynolds number boundary emerges. Below $R e_{2}$ of about 2000, all cases remained laminar and steady within the imaged region. This is a powerful result, as it lends confidence to the assumption that cases with lower Reynolds numbers could be computed using laminar CFD codes, irrespective of the JPR.

Although the unsteady data exhibit a great deal of scatter, a grouping of cases (orange squares) occurs at approximately two equivalent jet diameters. Upon closer investigation, these cases all consist of flows with a large barrel shock and Mach disk, and the location marked $x_{\text {unsteady }}$ occurs at the triple point and Mach disk of the flow. Unsteadiness is clearly originating from or being amplified by these points, as stated previously.

The other apparent trend in the supersonic data is the approximate boundary between the unsteady and transitional data. This boundary and the Reynolds number boundary dividing steady and unsteady flows have both been marked with dashed lines in Figure 14. Additionally, a power law fit to the fully transitional points is displayed, with an $R^{2}$ value of 0.76 . Only five cases in these tests were identified as becoming fully turbulent within the imaged region. Thus, no clear boundary can be established between transitional and turbulent cases. However, when the data are separated by $J P R$, additional trends are evident. Figure 15 illustrates these same data, but with jet pressure ratios greater than 3.5 indicated by open symbols. Note the clustering of data for the low JPR, non-Mach disk cases versus those at higher $J P R$ with a Mach disk. This reemphasizes the dependence on flow structure of the transition behavior of underexpanded jet flows.

Note that caution must be exercised in attempting to extrapolate these results to flows at conditions that fall outside those of the current data set. At high Reynolds numbers, the definitions of unsteady, transitional, and turbulent distances break down. This is because unsteadiness or even fully-developed turbulence may exist in the barrel-shock-processed gas along the jet boundary, while the accelerating flow inside the barrel shock is still steady and laminar. Peters and Phares ${ }^{18}$ report on such jets with a turbulent mixing layer along the jet boundary of the 
barrel shock. Krothapalli et al. ${ }^{11}$ and Zapryagaev et al. ${ }^{12}$ report streamwise vortices in the curved outer shear layer, which they attribute to a Taylor-Görtler instability, usually associated with flow along curved walls (described in Turbulent Flows, Pope $^{6}$ ).

\section{Conclusion}

PLIF images have been used to visualize underexpanded supersonic jet flows. These images have provided detailed information about flow structures and have allowed determination of the laminar, unsteady, transitional, or turbulent state of the flow. Additionally, the relationship between the axial distance to unsteady flow behavior and Reynolds number have been shown for supersonic nozzle cases.

Planar laser-induced fluorescence of nitric oxide has been successfully applied to the study of supersonic free jets, their flow structures, and the process of transition to turbulence. An image-processing technique was used to highlight flow instabilities by mapping the shot-to-shot percentage standard deviation in fluorescence intensity. These maps were used to quantitatively identify unsteady, transitional, and turbulent jet behavior. It was found that flows for which this rescaled Reynolds number was less than about 2,000 remained laminar and steady. All flows above this rescaled Reynolds number were found to become unsteady, in some cases transitioning to turbulent. A correlation was found between the rescaled Reynolds number and the distance to the onset of transition. Unsteady behavior was found to fall into groups based on whether the flows did have Mach disks (JPRs > 3.5) or had oscillating flow structures with no Mach disk $(J P R \mathrm{~s}<3.5)$.

Several normal modes of instability were observed. These included the sinuous and varicose axial modes, as well as cosine azimuthal modes. Standard deviation maps have helped to identify the flow features associated with the development of instabilities in these jet flows. Regions characterized by gradients in velocity-and therefore, non-zero vorticity - were found to be instrumental in the creation and amplification of flow instabilities. These included shock intersections (e.g. the triple point, the reflected shock/jet boundary intersection), shear layers (the outer shear layer along the jet boundary and the inner shear layer along the slip line emanating from the triple point), and locations of local jet diameter maxima (that is, local high-pressure antinodes) in flows with oscillating flow structures.

Volume imaging has been successfully demonstrated as an additional means of extracting flow visualization information from regions of the flow other than the flow centerline. In particular, the technique has been shown to effectively highlight departures from axisymmetric behavior. Volume imaging will also be useful in future experiments where limited optical access prevents imaging in the desired measurement plane.

These findings are being used by researchers at Boeing to help guide their computational approach to modeling underexpanded jet flows at conditions relevant to flight conditions of interest and/or specific damage scenarios. These studies ultimately help to inform the decision of whether repairs to the Shuttle Orbiter must be made prior to reentry. They have also demonstrated the richness of flow structure information that can be revealed by using a spatially resolved technique, as opposed to using a path-averaged line-of-sight technique like schlieren, and that resolving these features is crucial in obtaining insight into the complex process of transition to turbulence.

\section{Acknowledgments}

The authors wish to acknowledge the collaborative input of Scott Halloran of Rocketdyne, Don Picetti of The Boeing Company and Chris Glass of NASA Langley Research Center, as well as the technical assistance of Stephen Jones, and Paul Tucker, also of NASA Langley Research Center. They also wish to acknowledge the image processing work done by Aiyana Garcia, a graduate physics student from The College of William and Mary and a NASA GSRP (Graduate Student Researchers Program) student. This work was funded as part of the Shuttle Return to Flight effort through Chuck Campbell of Johnson Space Center and Tom Horvath of NASA Langley Research Center. Support was also received from the Aeronautics Research Mission Directorate's Fundamental Aeronautics Hypersonic Project, Experimental Capabilities discipline, under Robert Okojie. These tests were conducted in support of the Orbiter Aerothermodynamics Working Group as part of NASA's Shuttle Return to Flight (RTF) effort. 


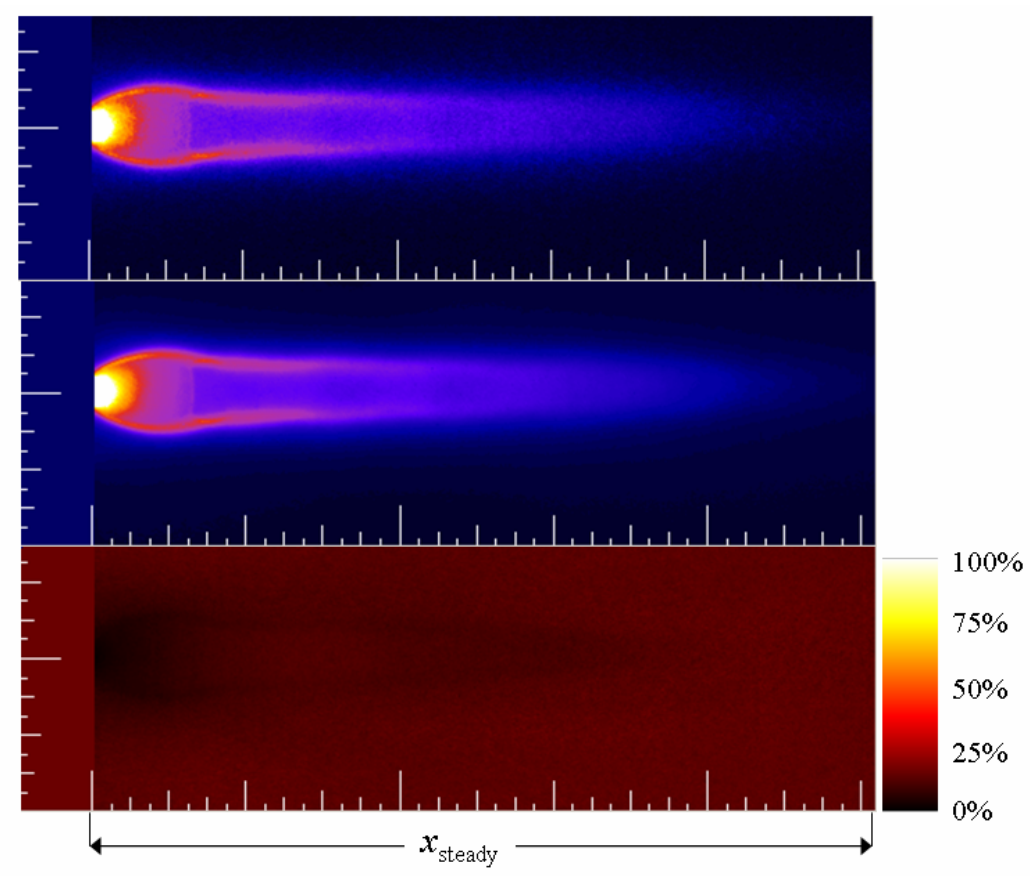

Figure 1: Image sequence for a steady laminar case (Run 49), with $J P R=15.8$ and $R e_{\text {exit }}=2644$. The top image is a single-shot image, the center image is the average of 100 single-shots, and the bottom image is a map of the percentage standard deviation in the image intensity at each pixel. The colorbar on the right indicates the color-scaling of the standard deviation image, with white corresponding to $100 \%$ variations in intensity, and black corresponding to no variation in intensity from shot-to-shot.

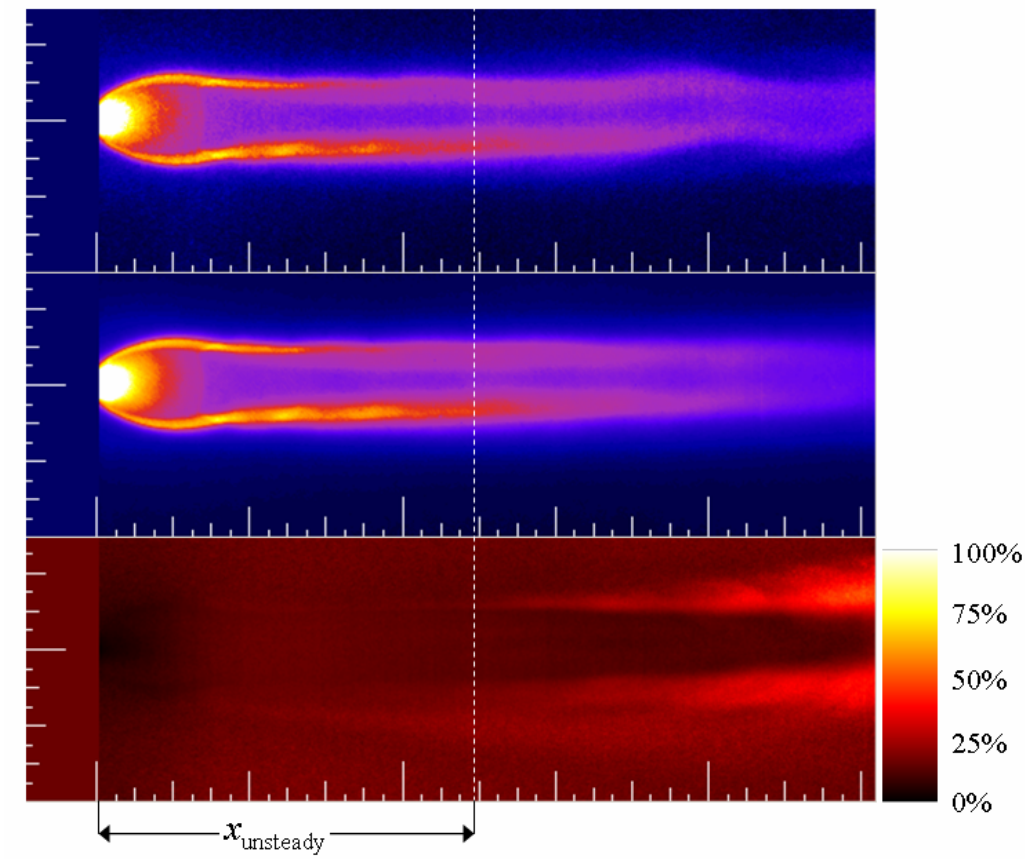

Figure 2: Image sequence for an unsteady laminar case ( $R$ un 35), with $J P R=16.3$ and $R e_{\text {exit }}=4435$. Notice the increase in intensity in the standard deviation image in the inner and outer shear layers. There may even be a small level of instability originating from jet boundary at the maximum jet diameter on the lower part of the image. 


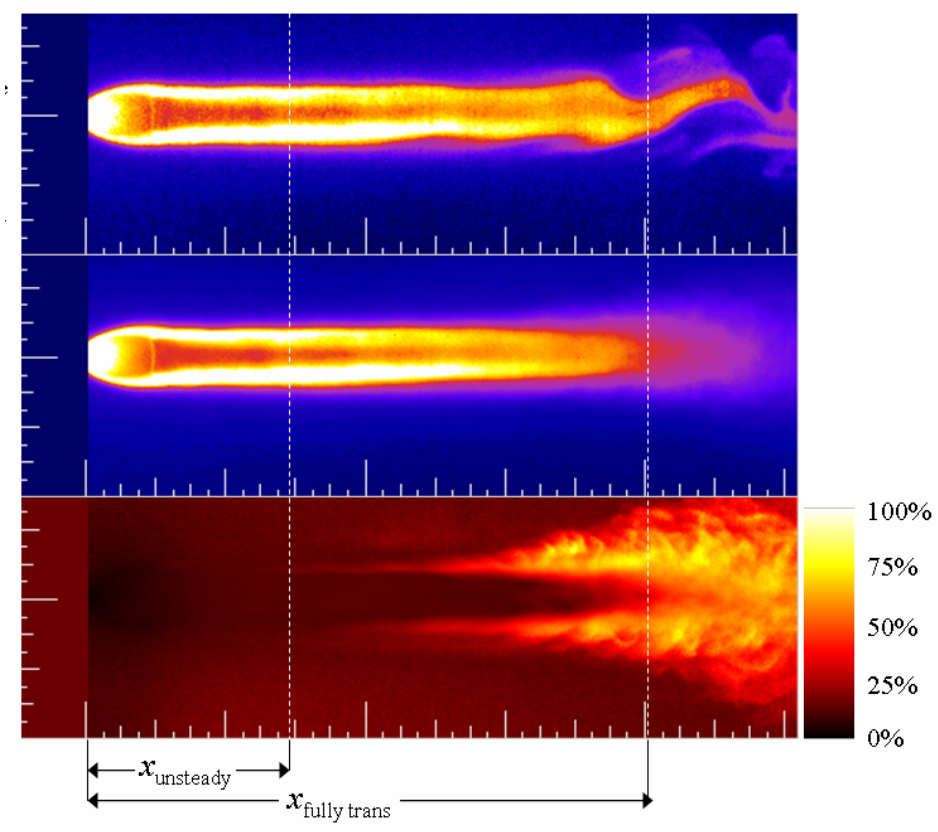

Figure 3: Image sequence for a fully transitional case (Run 31), with $J P R=8.7$ and $\operatorname{Re}_{\text {exit }}=4481$. Notice the initially slow growth of instabilities as the jet becomes unsteady, followed by the relatively fast growth of instabilities at the onset of transition. The fully transitional point is marked where the instabilities penetrate to the core of the jet.

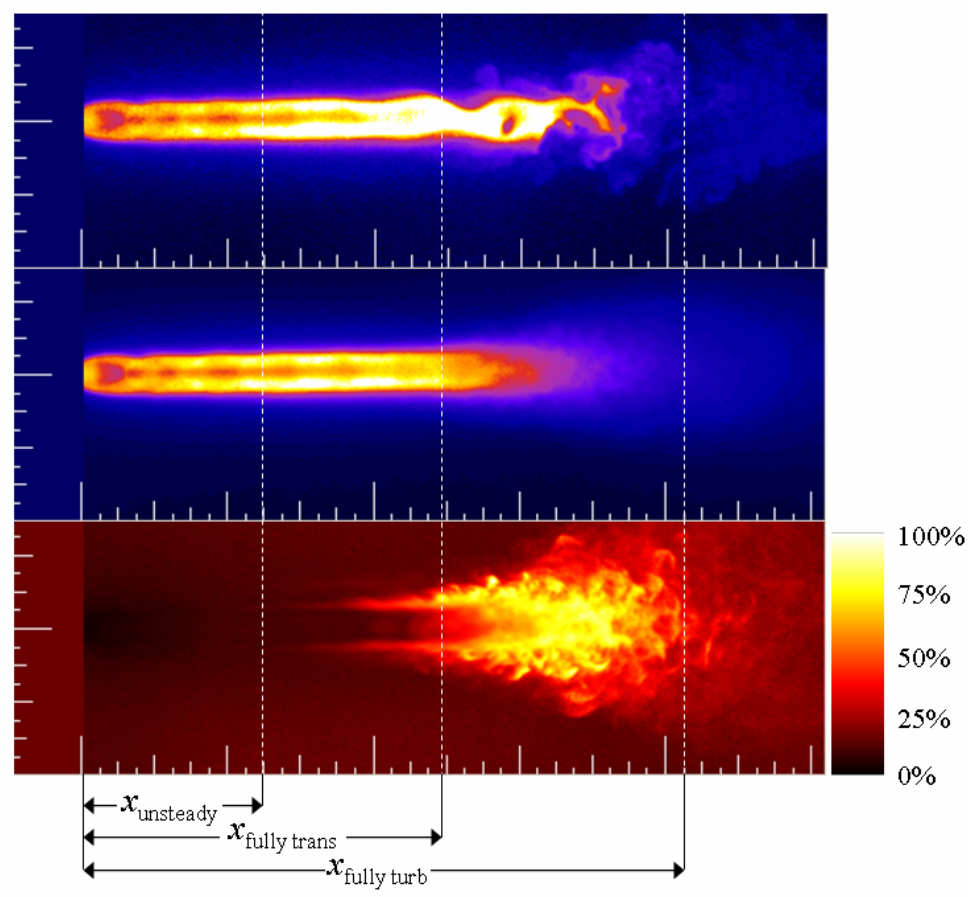

Figure 4: Image sequence for a fully turbulent case (Run 32), with $J P R=3.9$ and $\operatorname{Re}_{\text {exit }}=4460$. Notice the decrease in intensity in both the PLIF images (due to turbulent mixing and diffusion) and the standard deviation image (due to the homogeneous nature of turbulence) in the fully-developed region. The fully turbulent location is marked where the intensity in the standard deviation in the center of the flow decreases below the level it has in the transitional region.

American Institute of Aeronautics and Astronautics 092407 


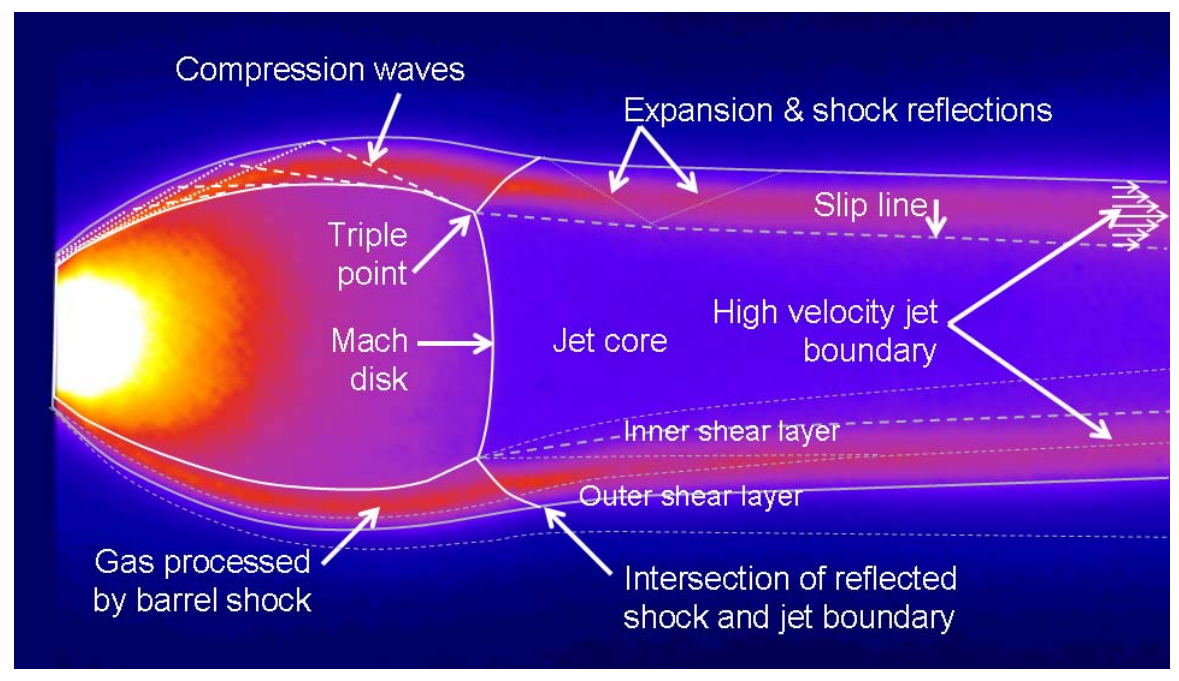

Figure 5. Highly -underexpanded sonic jet flow structures.
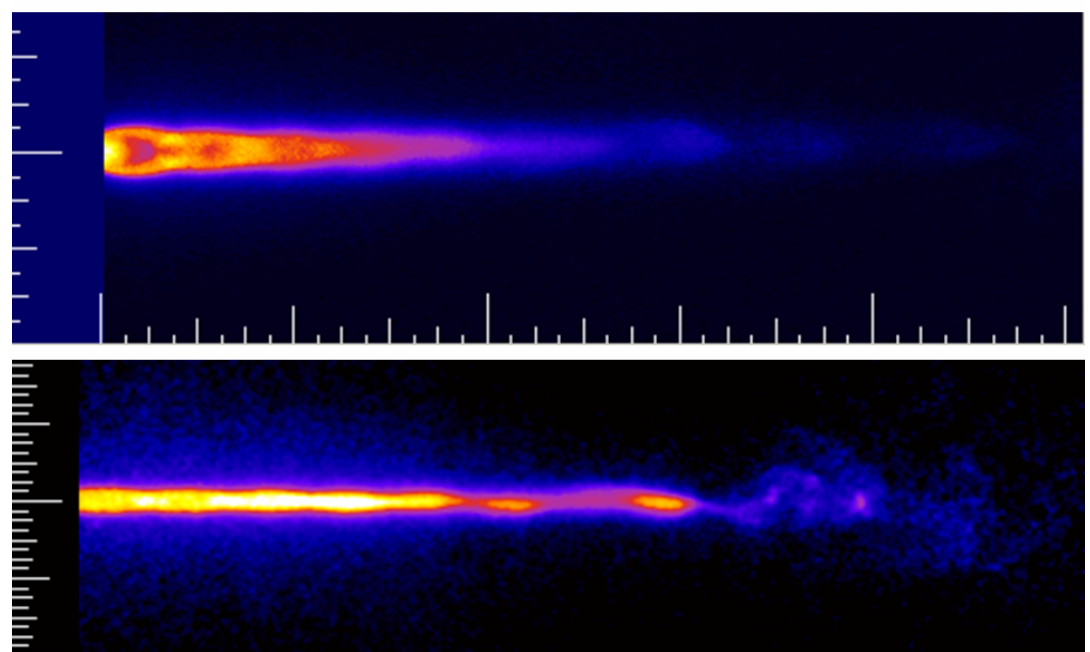

Figure 6. The varicose mode of unsteadiness. Both images are single-shot images. The upper image is a sonic nozzle flow (Run 46) $J P R=3.9$, and $R e_{\text {exit }}=2,593$; the lower image is a supersonic nozzle flow (Run 249) with JPR $=1.1$, and $R e_{\text {exit }}=6,698$. 

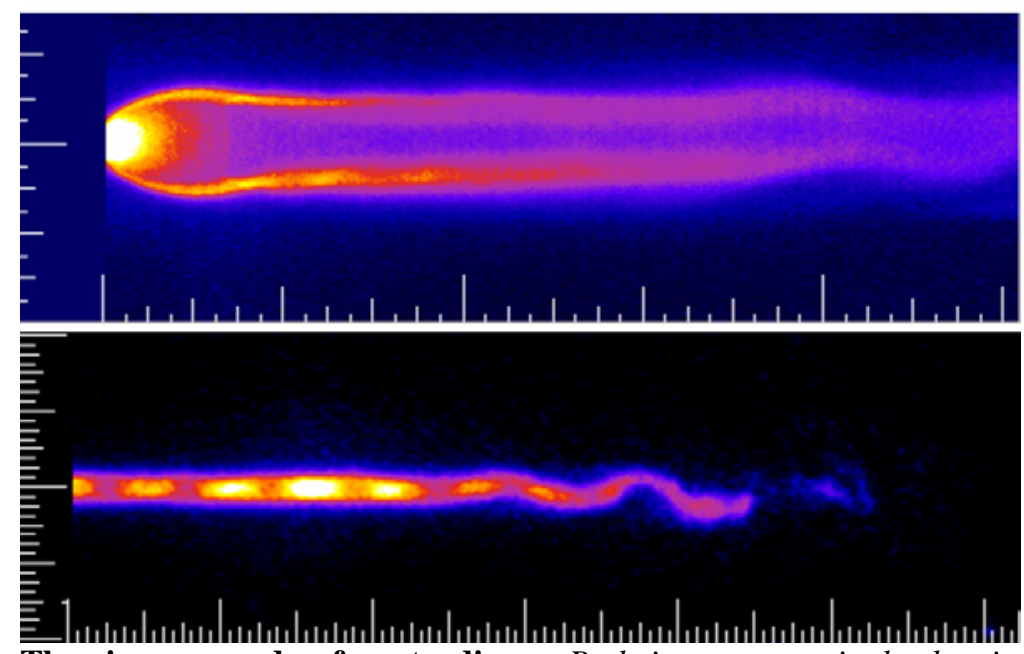

Figure 7: The sinuous mode of unsteadiness. Both images are single-shot images. The upper image is a sonic nozzle flow (Run35) $J P R=16.3$, and $R e_{\text {exit }}=4,361$; the lower image is a supersonic nozzle flow (Run 198) with $J P R=1.2$, and $R e_{\text {exit }}=9,064$.
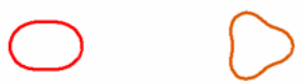

$\mathrm{m}=2$

$\mathrm{m}=3$

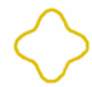

$\mathrm{m}=4$
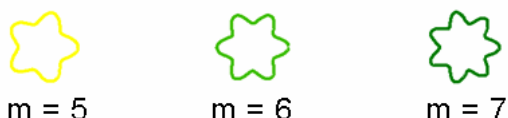

$m=5$

$m=6$

$\mathrm{m}=7$
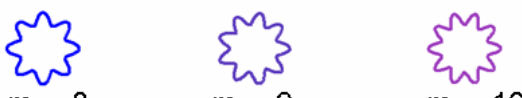

$\mathrm{m}=8$

$m=9$

$m=10$

Figure 8: Azimuthal $\cos (m \theta)$ modes for the first nine non-axisymmetric values of $\mathbf{m}$.

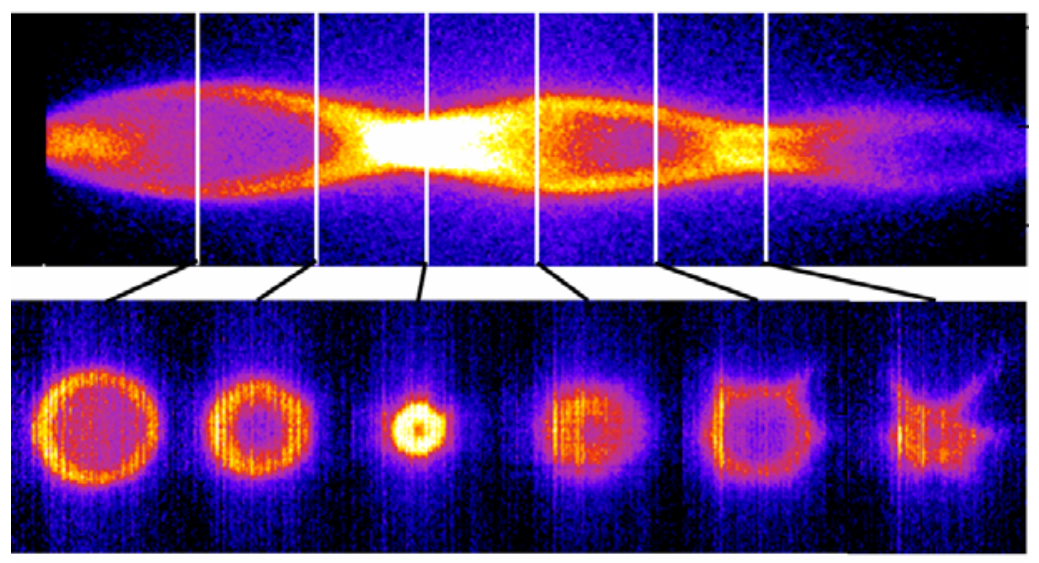

Figure 9: Six reconstructed slices and one single-shot centerline image. These images are from a supersonic nozzle run (Run 339) with $J P R=3.9$ and $R e_{\text {exit }}=4,303$. White vertical lines through the reference image indicate the axial locations of each of the slices. The fourth and fifth slices show evidence of the $\cos (m \theta)$ azimuthal instability mode, a feature not evident in the centerline image. Being able to capture symmetry-breaking flow features represents one of the advantages of volume-imaging.

American Institute of Aeronautics and Astronautics 092407 


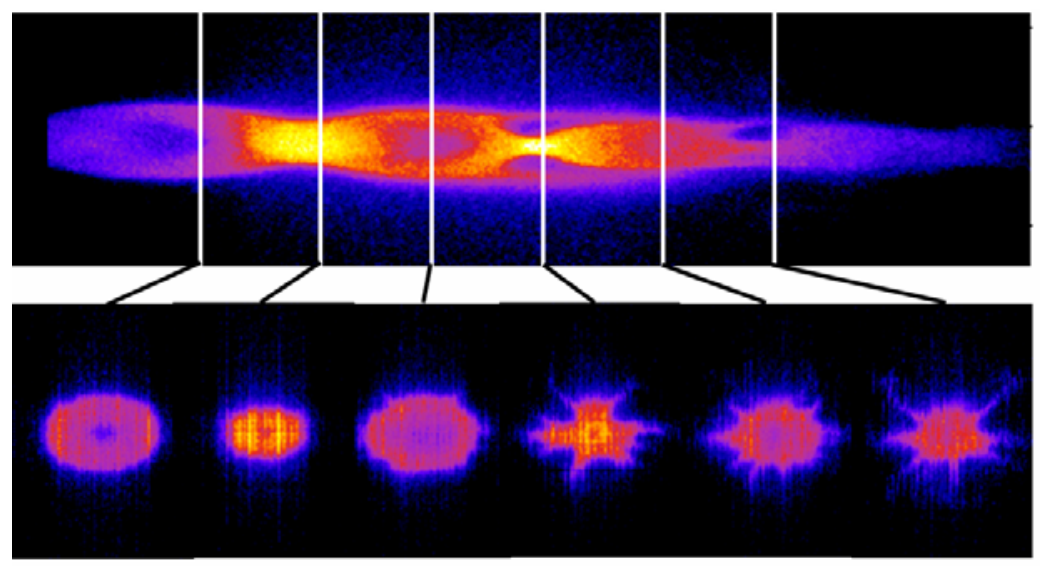

Figure 10: Six reconstructed slices and one single-shot centerline image. These images are from a supersonic nozzle run (Run 342) with $J P R=2.1$ and $R e_{\text {exit }}=9,501$. White vertical lines through the reference image indicate the axial locations of each of the slices. Note the growth of azimuthal instability modes stemming from the second flow maximum. The fact that the cross-sectional slices are constructed from 200 single shots (which take 20 seconds to acquire) indicates that the breakdown mode shown here is relatively persistent and spatially stable.
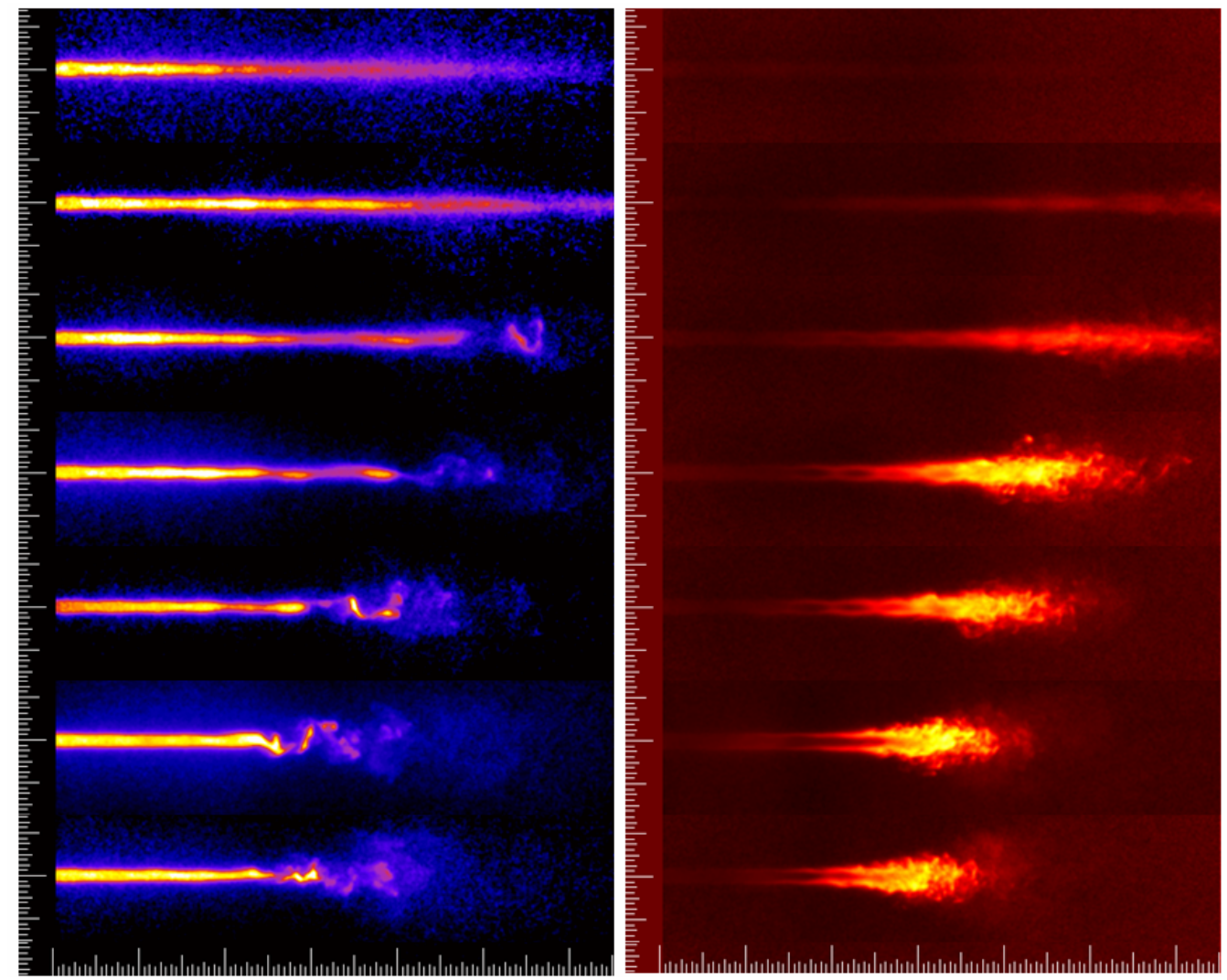

Figure 11: The effect of Reynolds number on transition to turbulence for a jet pressure ratio of about 1.1. Single-shot PLIF images from supersonic nozzle runs (left) are displayed along with the corresponding standard deviation maps (right) for six values of $\operatorname{Re}_{2}$ (top to bottom): 2,045; 3,383; 4,510; 6,$540 ; 7,875 ; 10,593 ; 13,039$. Smallest hash marks measure $1 / 16^{\text {th }}$ of an inch. (For reference, these are Runs 256, 227, 228, 249, 251, 221, 252).

American Institute of Aeronautics and Astronautics 092407 


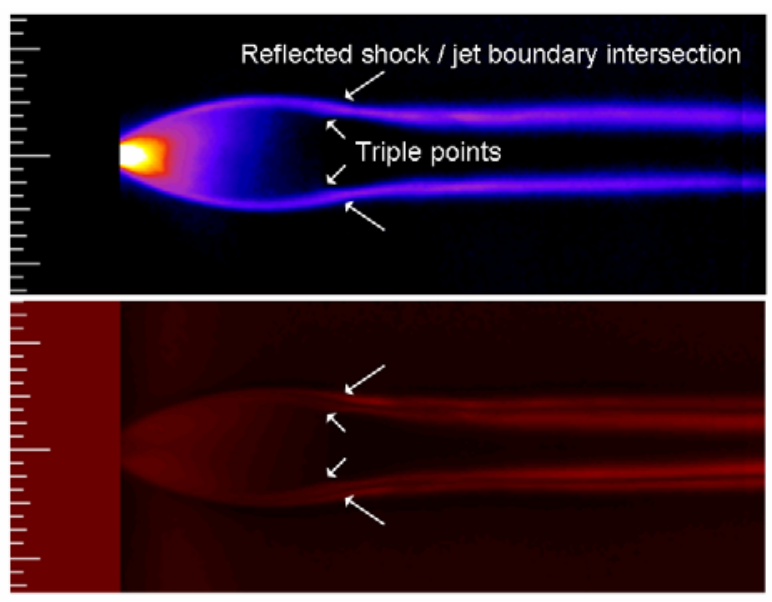

Figure 12: Triple point and reflected shock/jet boundary intersection as sources of instability. The upper image is a single-shot PLIF image of a supersonic nozzle flow (Run 650) with JPR $=19.2$, and $R e_{\text {exit }}=17,240$. The lower image is a standard deviation image. Note how instabilities in the inner and outer shear layers that border the high-velocity jet boundary stem back to the triple points and the reflected shock/jet boundary intersections. Smallest hash marks measure $1 / 16^{\text {th }}$ of an inch.
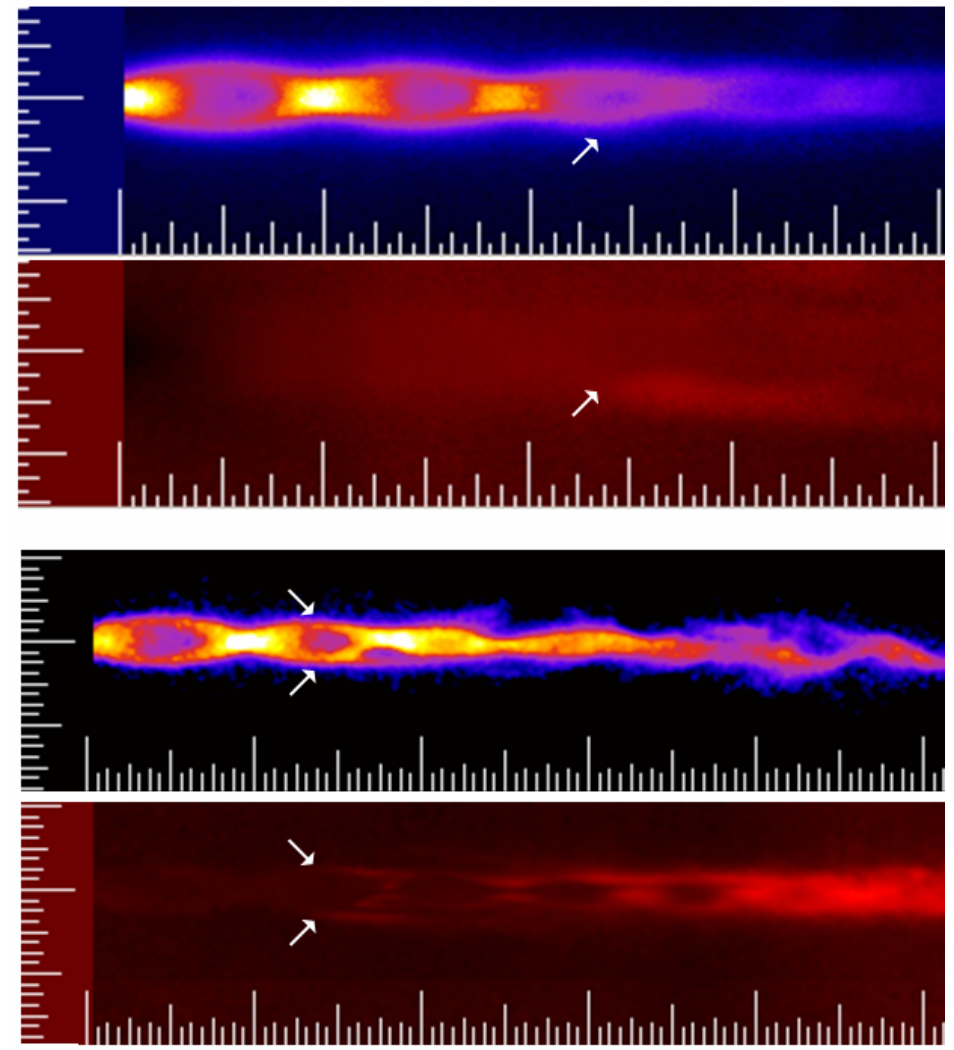

Figure 13: Flow maximum as a source of instability. The upper image is a single-shot PLIF image from a supersonic nozzle run (Run 143) with $J P R=3.4, R e_{\text {exit }}=4,269$. Note the beginnings of instability emanating from the third flow maximum on the bottom of the image. The lower image is from a run (Run 246) with a similar JPR (3.1) but at a higher exit Reynolds number (8,001). The standard deviation image highlights instabilities stemming from the top and bottom of the second flow maximum. 


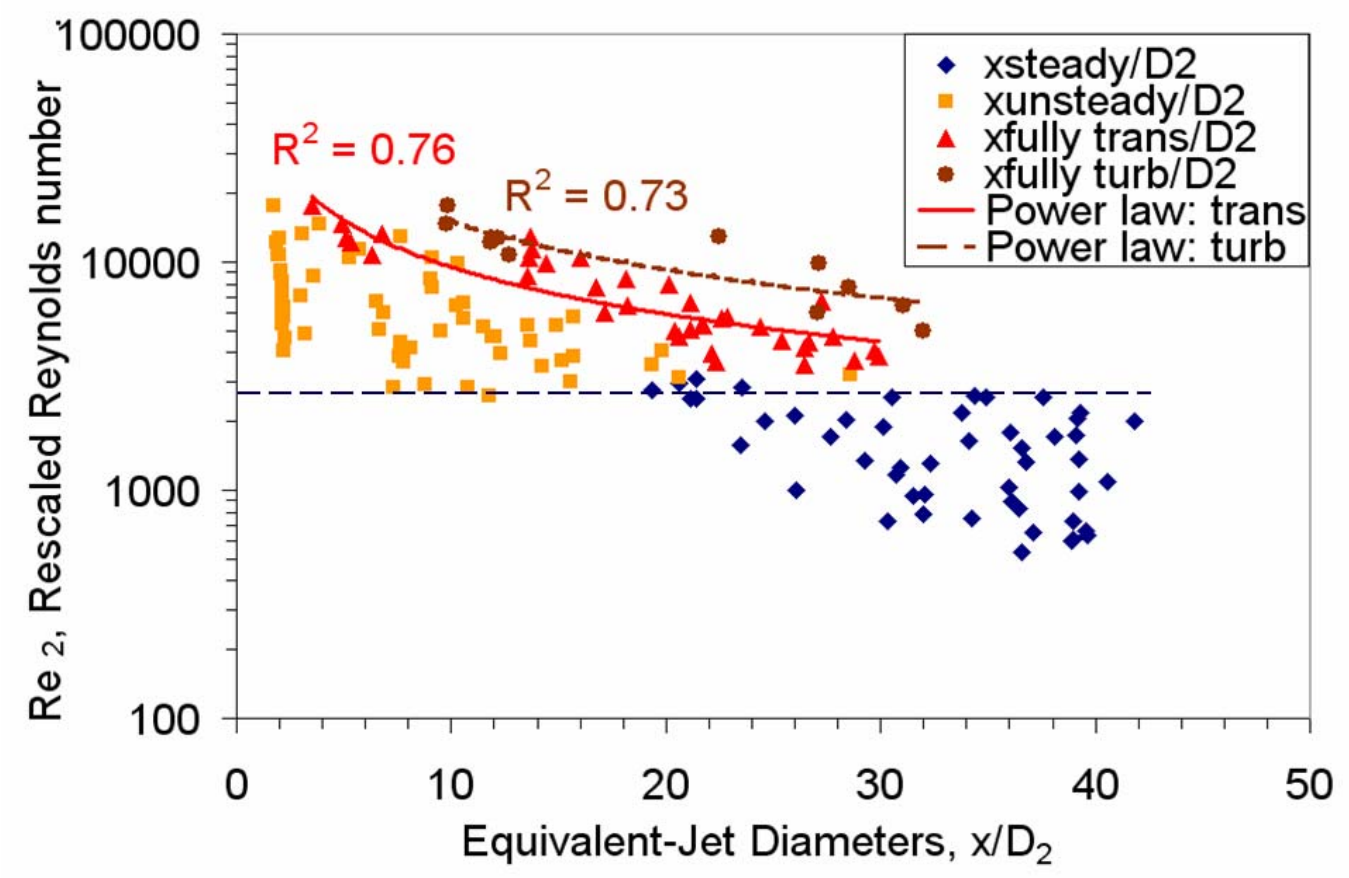

Figure 14: Transition correlation for supersonic free jet cases. A dashed blue line marks the boundary between steady and unsteady flow at a rescaled Reynolds number of 2600. A solid red line shows a power-law fit to the data, with a resulting $R^{2}$ value of 0.76 , and a dashed brown line shows a power law fit to the turbulent data, with an $R^{2}$ value of 0.73 .

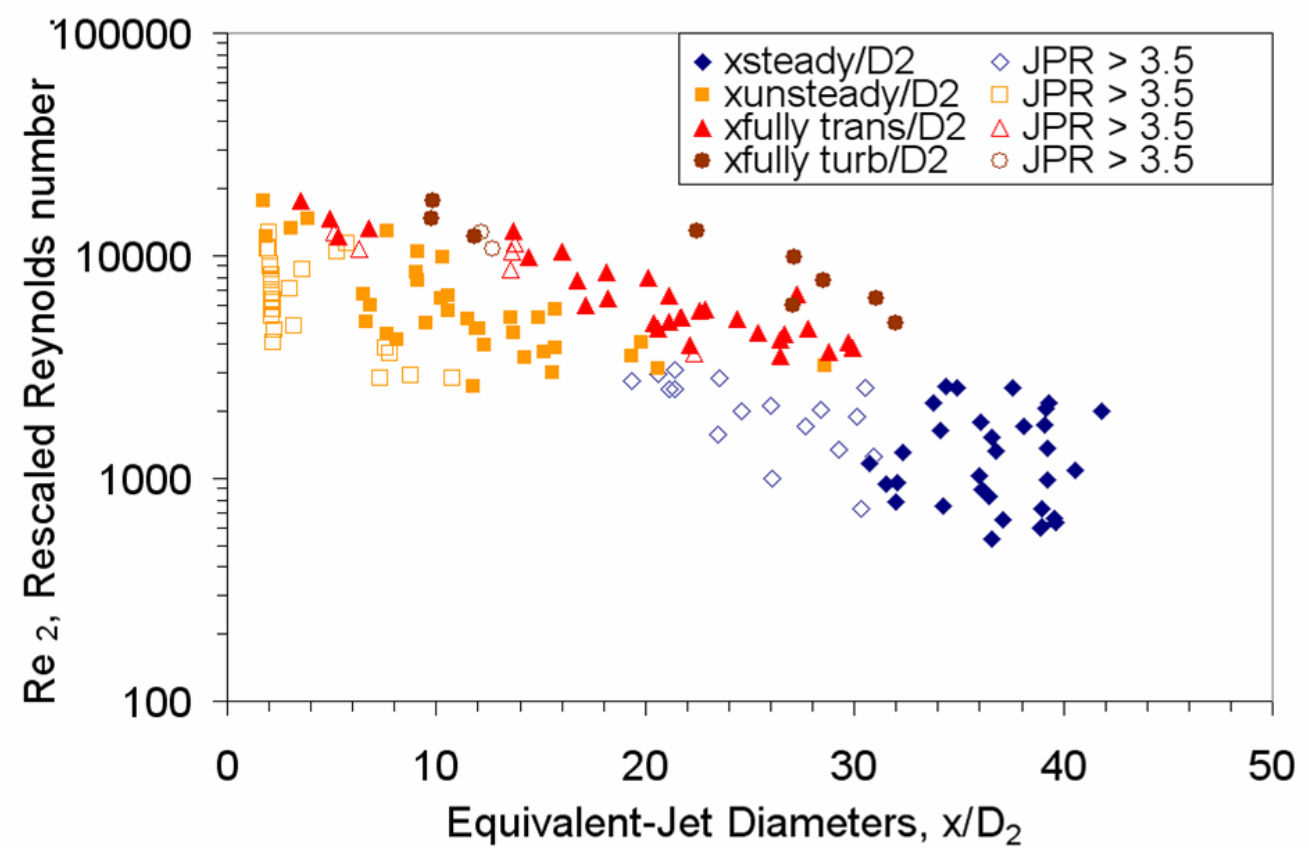

Figure 15: Supersonic free jet cases, with data separated by jet pressure ratios above 3.5 (open symbols) and below 3.5 (solid symbols). 


\section{References}

\footnotetext{
${ }^{1}$ Wilkes, Jennifer A., Paul M. Danehy, and Robert J. Nowak "Fluorescence Imaging Study of Transition in Underexpanded Jets," Proceedings of the $21^{\text {st }}$ International Congress on Instrumentation in Aerospace Simulation Facilities (ICIASF) [CDROM], Sendai, Japan, 29 August - 1 September 2005, pp. 1-8.

2 Wilkes, Jennifer A., Christopher E. Glass, Paul M. Danehy, and Robert J. Nowak, "Fluorescence Imaging of Underexpanded Jets and Comparison with CFD," AIAA 2006-0910, $44^{\text {th }}$ AIAA Aerospace Sciences Meeting and Exhibit, Reno, NV, 9-12 January 2006.

${ }^{3}$ Inman, Jennifer A. (Wilkes), Paul M. Danehy, Robert J. Nowak, and David W. Alderfer, "Fluorescence Imaging Study of Impinging Underexpanded Jets," AIAA 2008-619, 46 th AIAA Aerospace Sciences Meeting and Exhibit, Reno, NV, 7-10 January 2008.

${ }^{4}$ Anderson, J. D., Jr. (2003). Modern Compressible Flow: With Historical Perspective, $3^{\text {rd }}$ ed., McGraw-Hill, Boston.

${ }^{5}$ Inman, Jennifer A., "Fluorescence Imaging Study of Free and Impinging Supersonic Jets: Jet Structure and Turbulent Transition,” Ph.D. Dissertation, Department of Physics, The College of William and Mary, Williamsburg, VA, 2007.

${ }^{6}$ Pope, Stephen B. Turbulent Flows. Cambridge University Press, Cambridge, U.K. (2000).

${ }^{7}$ Drazin, P.G., and W.H. Reid (1981). Hydrodynamic Stability, edited by G.K. Batchelor and J.W. Miles, Cambridge University Press, Cambridge, U.K.

${ }^{8}$ Lesieur, M. Turbulence in Fluids: Stochastic and Numerical Modeling. Martinus Nijhoff Publishers, Dordrecht, The Netherlands (1987).

${ }^{9}$ Donaldson, C. D., and R. S. Snedeker (1971). "A study of free jet impingement. Part 1. Mean properties of free and impinging jets," J. Fluid Mech. 45, Part 2: 281-319.

${ }^{10}$ Donaldson, C. D., R. S. Snedeker, and D. P. Margolis (1971). "A study of free jet impingement. Part 2. Free jet turbulent structure and impingement heat transfer," J. Fluid Mech. 45, Part 3: 477-512.

${ }^{11}$ Krothapalli, A., G. Buzyna, and L. Lourenco (Aug 1991). "Streamwise vortices in an underexpanded axisymmetric jet," Phys. Fluids A 3 (8): 1848-1851.

${ }^{12}$ Zapryagaev, V., Pickalov, V., Kiselev, N., and Nepomnyashchiy, A. "Combination interaction of Taylor-Goertler vortices in a curved shear layer of supersonic jet," Theoretical and Computational Fluid Dynamics 18 (2-4) pp. $301-308$ (November 2004).

${ }^{13}$ Danehy, P.M., A.P. Garcia, S. Borg, A.A. Dyakonov, S.A. Berry, J.A. (Wilkes) Inman, and D.W. Alderfer (8-11 Jan 2007). "Fluorescence visualization of hypersonic flow past triangular and rectangular boundary-layer trips." $45^{\text {th }}$ AIAA Aerospace Sciences Meeting and Exhibit, Reno, NV. AIAA 2007-0536.

${ }^{14}$ Tipler, P.A. (1990). Physics for Scientists and Engineers, $3^{\text {rd }}$ ed. Worth Publishers, New York.

${ }^{15}$ Balakumar, Ponnampalam (Jan 2007), Flow Physics and Control Branch, NASA Langley Research Center, Hampton, VA. Private communication.

16 van Cruyningen, Ike (Sep 1990). Quantitative Imaging of Turbulent Gaseous Jets Using Planar Laser-Induced Fluorescence. M.S. thesis, High Temperature Gasdynamics Laboratory (HTGL), Stanford University, Stanford, CA. HTGL Report No. T-267.

${ }^{17}$ Yüceil, K. Bülent, and M. Volkan Ötügen (Dec 2002). "Scaling parameters for underexpanded supersonic jets," Physics of Fluids 14, (12): 4206-4215.

${ }^{18}$ Peters, C.E., and Phares, W.J. "The structure of plumes from moderately underexpanded supersonic nozzles," $8^{\text {th }}$ AIAA Aerospace Sciences Meeting, AIAA 70-229, New York, NY, 19-21 January 1970.
} 